\title{
Evaluation of feasibility, efficiency and safety of a pure NOTES gastrojejunal bypass with gastric outlet obstruction, in an in vivo porcine model
}

Authors

Institutions
J.-M. Gonzalez ${ }^{1,2}$, E. A. Bonin ${ }^{1}$, G. Vanbiervliet ${ }^{3}$, E. Garnier ${ }^{4}$, S. Berdah ${ }^{5}$, K. Matthes ${ }^{2}$, M. Barthet ${ }^{1}$

Institutions are listed at the end of article. received 23. May 2013 accepted after revision 5. December 2013

\section{Bibliography}

Dol http://dx.doi.org/ 10.1055/s-0033-1353686 Endoscopy International Open 2013; 1: E31-E38 (c) Georg Thieme Verlag KG Stuttgart $\cdot$ New York E-ISSN 2196-9736

Corresponding author Dr. Jean-Michel Gonzalez, MD Laboratory Dana 506 Department of Gastroenterology Beth Israel Deaconess Medical Center

Harvard Medical School

Boston, MA

United States jmgonza05@yahoo.fr jgonza11@bidmc.harvard.edu
Introduction: Natural orifice transluminal endoscopic surgery (NOTES) gastrojejunal anastomosis (GJA) is a less invasive surgery for bariatric procedures and gastric outlet obstruction. The aim of this study was to evaluate the feasibility, efficacy, and safety of a pure NOTES gastrojejunal bypass using an in vivo porcine model.

Material and methods: A prospective study was performed on nine swine. A double-channel scope was used. The intervention steps were: (i) gastric incision; (ii) peritoneal access; (iii) jejunal loop selection and mobilization into the stomach; (iv) stoma creation within the gastric wall and incision; (v) anastomosis suture and pylorus closure using a T-tag prototype. The animals were assessed clinically for 3 weeks including the weight gain. The patency of the GJA was assessed at necropsy and a histological analysis was performed.

\section{Introduction}

Natural orifice transluminal endoscopic surgery (NOTES) is an emerging technique for accessing the peritoneum without parietal incisions in an effort to potentially reduce procedure-related morbidity and mortality $[1,2]$. Although the outcome of laparoscopic gastrojejunal anastomosis (GJA) appears to be improving, it is still associated with surgical and anesthetic adverse events, including anastomotic insufficiency, ventral hernia, and wound infections [3-10]. NOTES may present a less invasive alternative for GJA for various indications, including benign or malignant gastroduodenal outlet obstruction and bariatric procedures. Only a few studies have assessed the feasibility of performing this procedure exclusively by NOTES with, to date, 42 GJA procedures reported in the literature in the porcine survival animal model. These reports are associated with some limitations, including small sample sizes, lack of reproducibility, and absence of histological assessment. Moreover, virtually all survival studies
Results: We successfully performed all the procedures with a mean (standard deviation [SD]) operative time of 108 (26) minutes. We used a mean of 5.55 (1.30) stitches. There were no intraprocedural adverse events. Five animals survived up till euthanasia at 3 weeks (65\%). These showed a significant difference in weight curves of a loss of $3.2 \mathrm{~kg}$ compared with gain of $5.2 \mathrm{~kg}$ in a control group.Four pigs died from anastomotic dehiscence complicated by peritonitis.

Conclusion: Gastrojejunal bypass with a pure NOTES approach is feasible. This procedure is effective, resulting in a patent anastomosis and a significant weight loss. However, the anastomotic dehiscence is a major concern because of its mortality rate, and further studies including improvement of the suturing device and the technique are needed.

using animal models were performed as laparoscopically assisted ("hybrid") NOTES procedures [11-18]. The creation of a pure NOTES bariatric gastric bypass appears to be technically difficult in human cadavers $[19,20]$.

Several endoscopic tissue approximation systems have been proposed for the closure of the gastrostomy after NOTES, including T-tags, Padlock-G clip (Aponos Inc.), and the "over-the-scope" system (OVESCO) [21-25].

The aim of this study was to evaluate the feasibility, safety, and efficacy of a pure NOTES gastrojejunal bypass procedure in a 3-week survival porcine model. Feasibility and efficacy were assessed using technical parameters such as procedure time, technical difficulty, and the occurrence of intraoperative adverse events. Safety was assessed by monitoring the animals postoperatively for any clinical signs of intra-abdominal infection and sepsis. Anastomotic integrity and patency were evaluated grossly during necropsy as well as histologically. The clinical outcome was evaluated by monitoring dietary intake and by weight curve assessment. 


\section{Materials and methods}

\section{Study design}

This was a prospective experimental survival animal study conducted at the Center for Surgical Education and Research (CERC) of the Faculty of Medicine North, Mediterranean Aix-Marseille University. Institutional review board approval was obtained prior to conducting the study.

The experimental protocol consisted of performing a purely endoscopic procedure with general anesthesia on nine consecutive healthy young domestic Yorkshire "minipigs" of either sex, aged 3-4 months and weighing between 25 and $30 \mathrm{~kg}$. All animals were allowed to recover and were then clinically observed under normal feeding and housing conditions. Euthanasia and necropsy were performed following a 3-week survival period.

\section{Animal preparation, anesthetic and analgesic protocols} All animals were fasted for 24 hours prior to intervention. Anesthesia was induced by an intramuscular injection of $120 \mathrm{mg}$ of azaperone (Stresnil) coupled with $70 \mathrm{mg}$ of ketamine, followed by endotracheal intubation. Anesthesia was maintained by continuous intravenous infusion of propofol at a rate of $100 \mathrm{mg}$ per hour (Diprivan 2\%), and fentanyl was given at a dose of $100 \mathrm{mi}-$ crograms per hour for analgesia, with monitoring of heart rate and oxygen saturation via pulse oximetry. The animals also received $1 \mathrm{~g}$ of cefotaxime as antimicrobial prophylaxis preoperatively as well as intraoperatively. All animals were placed in a supine position for the procedure.

Following recovery from anesthesia, pain was assessed twice a day by observing animal behavior, and $1 \mathrm{~g}$ of cefotaxime was administered intramuscularly daily for a period of 7 days.

\section{Endoscopic equipment and intervention stages}

All procedures were carried out using a double-channel video esophagogastroduodenoscopy endoscope (3.8-and 2.8-mm channel diameters; Karl Storz GmbH \& Co. KG, Tuttlingen, Germany). The electrosurgical unit used was the Olympus ESG-100 (Olympus Corporation, Tokyo, Japan). Three interventional endoscopists performed all the procedures.

The NOTES GJA was performed using a standardized method. All the stomachs were decontaminated before each intervention. To access the peritoneum, a longitudinal, plane-by-plane gastric incision of $3-5 \mathrm{~cm}$ was made using the needle-knife (Olympus Europe Corporation) at the anterior wall of the stomach in the preantral region ( $\bullet$ Fig. $\mathbf{1}$ ). A pneumoperitoneum was created using room air insufflation. A jejunal loop was selected and grasped on its border with a twin-grasping forceps (Twin Grasper; OVESCO AG, Tuebingen, Germany). The optimal loop was selected after peritoneal examination, based only on its proximity to the access site to avoid further tension on the anastomosis. The selected loop was mobilized ( $\boldsymbol{O}$ Fig. 2 ) toward the gastric incision site with careful attention to avoiding tension on the loop that might possibly result in anastomotic dehiscence or bowel ischemia postoperatively.

To create the stoma, the mesentery under the raised loop was punctured and the rigid end of a 0035" guidewire (Jagwire Stiff; Boston Scientific Corporation, Natick, USA) was advanced within a sphincterotome (Tritome; Cook Medical, Limerick, Ireland) and through the mesentery ( $\mathbf{F i g . 3}$ ). The sphincterotome was then exchanged for placement of a fully covered self-expandable metallic (SEMS) biliary stent $(10 \mathrm{~cm}$ long, CLBS; Cook Medical, Limerick, Ireland) with its free ends being within the mesentery on

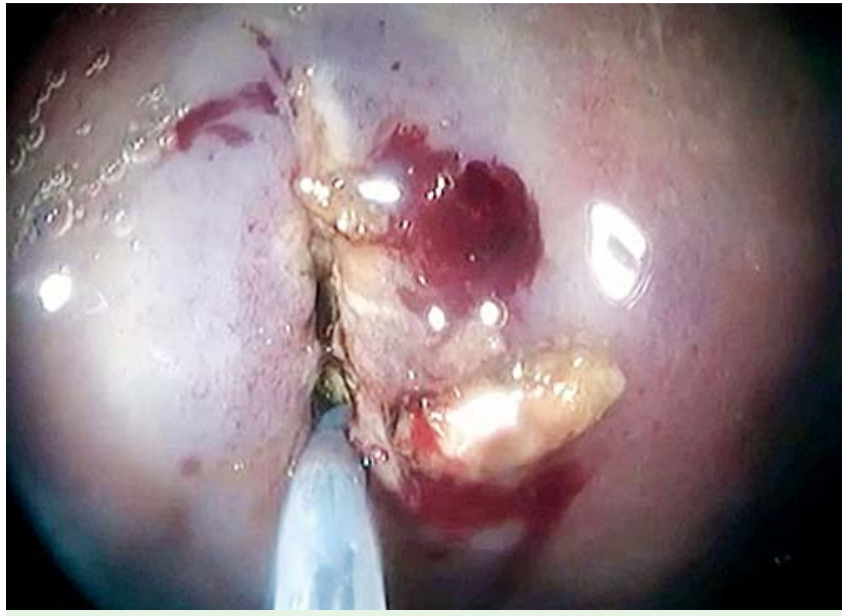

Fig. 1 Longitudinal, plane-by-plane gastric incision using the needle-knife at the anterior wall of the stomach in the pre-antral region.

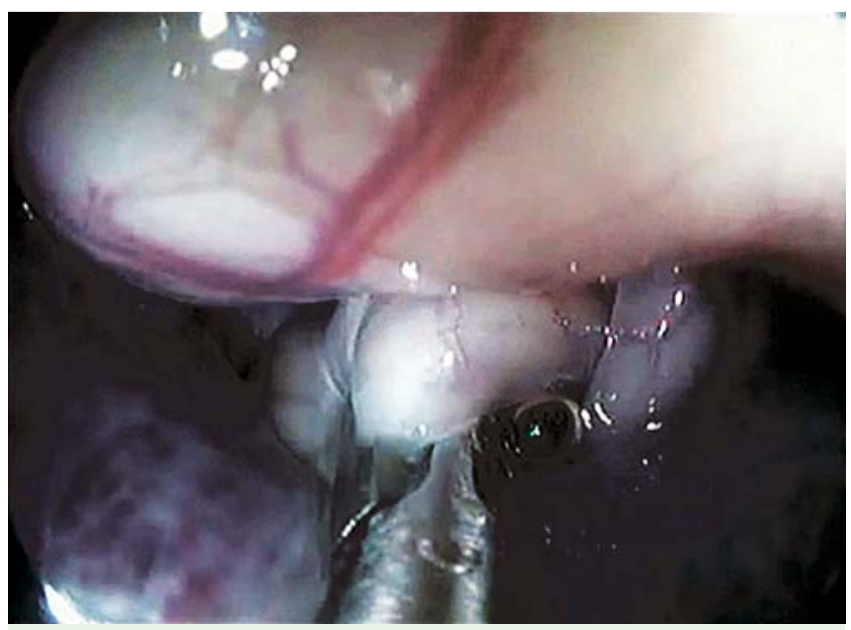

Fig. 2 Selection, grasping and mobilization of an appropriate jejunal loop toward the gastric incision with a Twin-Grasper forceps.

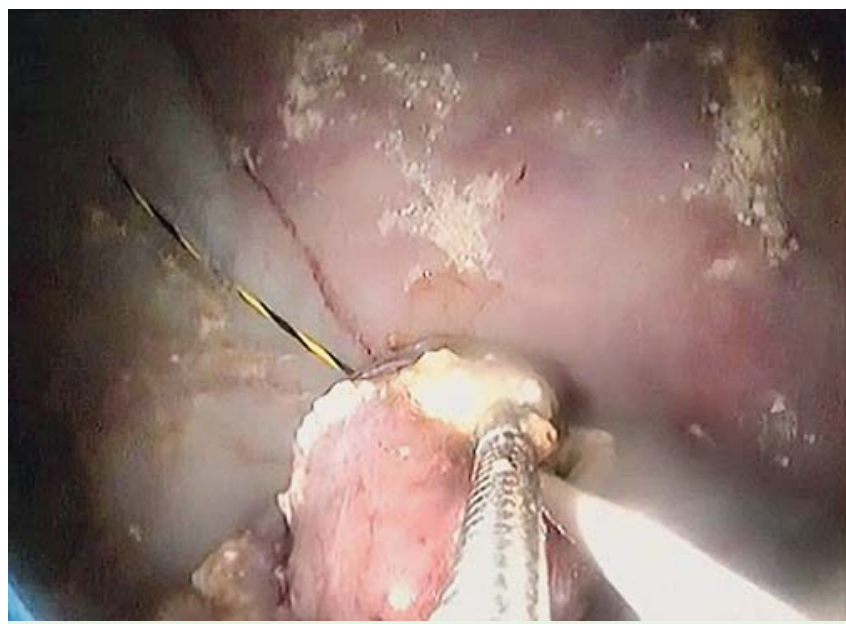

Fig. 3 Puncture of the mesentery under the raised loop with the rigid end of a $0035 "$ guidewire, which was then advanced within a sphincterotome and through the mesentery. 


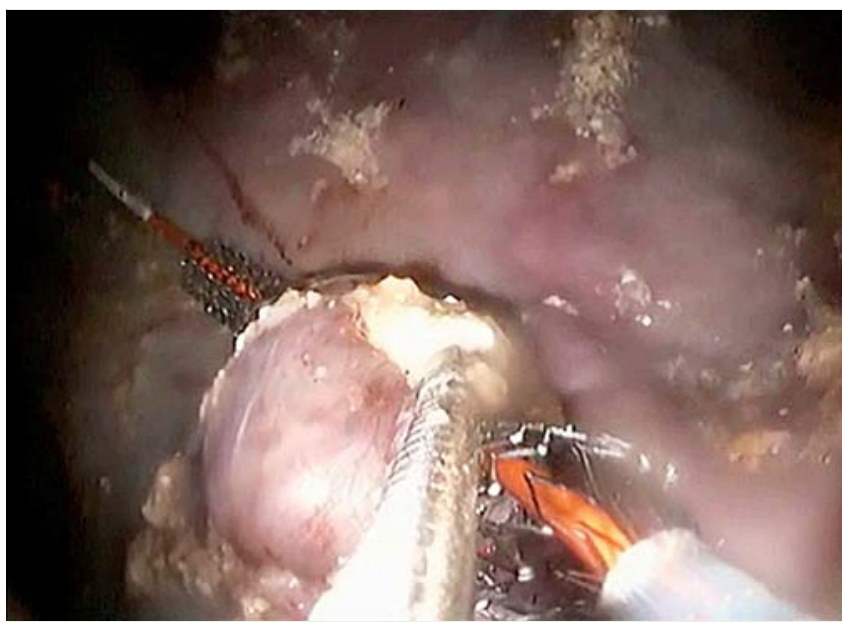

Fig. 4 Placement of a fully-covered biliary self-expandable metallic stents (SEMS) $(10 \mathrm{~cm}$ length), with its free ends being within the mesentery on both sides of the loop.

both sides of the loop ( $\bullet$ Fig.4). This provided the same function as a loop ileostomy rod, preventing the intestinal loop from falling into the peritoneal cavity when the stoma was created. The jejunum was opened with a longitudinal 2-cm incision at the antimesenteric border, using a Dual Knife, and then extended with a Hook Knife (Olympus Corporation).

Full-thickness suturing of the jejunal loop into the gastric stoma was accomplished using a Brace Bar prototype suturing system ( $\bullet$ Fig.5) (Brace Bar; Olympus, Japan). This device consists of a needle with suture thread within the sheath and two T-tags, one on each end of the thread. For creating the anastomosis, two sutures were dropped successively through the jejunal and gastric walls. The two suture sides were then secured with a crimping device that tightens the two stitches against one another. A complete anastomosis was achieved using four to seven stitches equally distributed along the anastomotic perimeter ( $\bullet$ Fig. $\mathbf{6}$ ). The biliary SEMS was removed using the Twin Grasper followed by placement of two extra T-tag sutures to close the gaps left by the stent. The anastomotic integrity was finally carefully inspected, visually and using the grasper, to detect any gaps between sutures. We could not fill the stomach with contrast because fluoroscopy was not available.

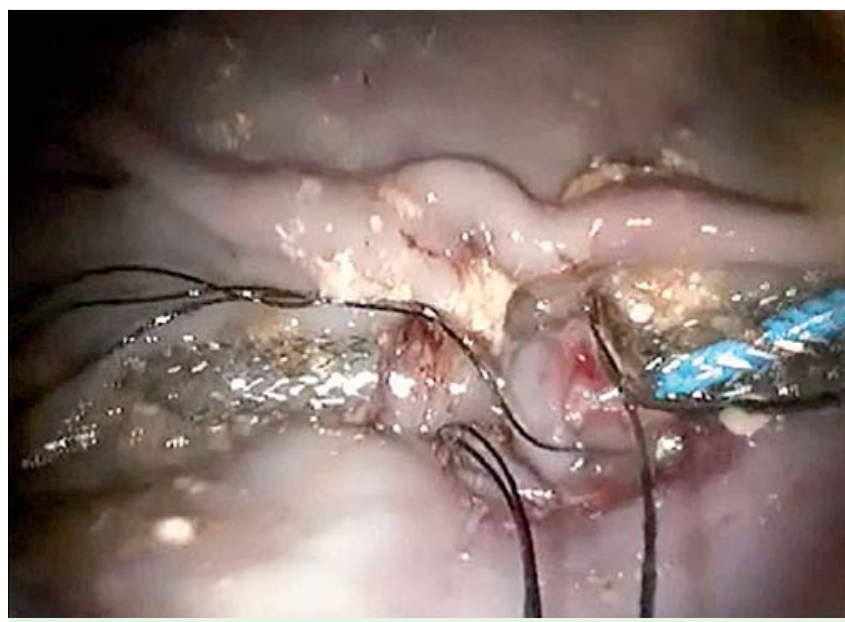

Fig. 6 Achievement of the complete anastomosis using four to seven stitches equally distributed along the anastomotic perimeter.

To simulate a gastric outlet obstruction, two T-tags were placed at the level of the pylorus in an " $\mathrm{X}$ " fashion. Following a final inspection, the stomach was decompressed and the endoscope withdrawn. In the case of bleeding, coagulation was attempted using a coagulation forceps (Coagrasper, Olympus Corporation, Japan) set at $60 \mathrm{~W}$ in the soft-coagulation mode (Olympus ESG-100 (Olympus Corporation, Tokyo, Japan). In the case of signs of tension pneumoperitoneum, decompression of insufflation was accomplished using a percutaneously placed 20G needle.

\section{Follow-up and postoperative protocol}

All animals were clinically observed for a period of 3 weeks. After recovery from anesthesia, animals were kept fasted with access to water until the third postoperative day (POD). Institution of nutrition was performed gradually: a quarter of the usual feeding was given for 48 hours with progression to half portions for 48 hours before normal feeding from POD 3 until POD 21.Clinical follow-up was performed daily, with twice-daily monitoring of overall behavior, food intake, fever, pain, and bowel and urinary function. The animals' weights were measured at baseline and at the end of the 3-week study period.

The weight curve of a control group of the same number of animals with the same characteristics (3-4 months old) was also followed for 3 weeks. These animals were enrolled in a study
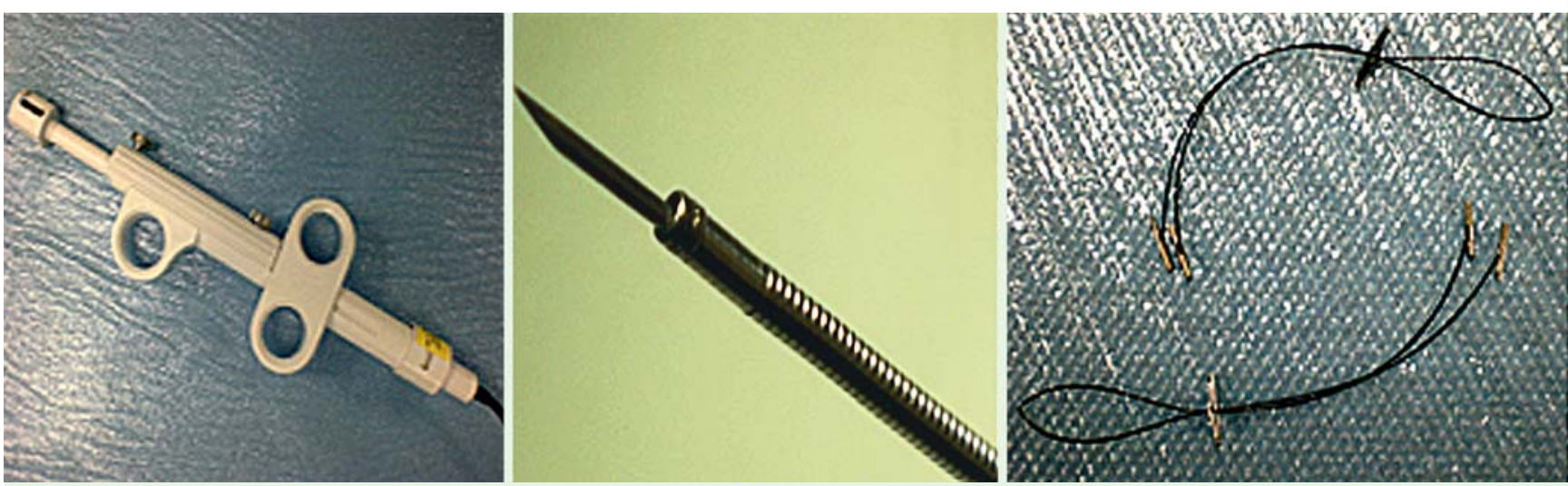

Fig. 5 The Brace Bar suturing system. Needle, handle, and T-tag recharge. 
Table 1 Summary of technical and clinical outcomes from natural orifice transluminal endoscopic surgery (NOTES) gastrojejunal bypass in nine pigs

\begin{tabular}{|c|c|c|c|c|c|c|c|c|c|}
\hline & \multicolumn{9}{|c|}{ Animal no. } \\
\hline & 1 & 2 & 3 & 4 & 5 & 6 & 7 & 8 & 9 \\
\hline \multicolumn{10}{|l|}{ Anesthesia } \\
\hline Oxygen rate $<90 \%$ & No & No & No & No & No & No & No & No & No \\
\hline Tachycardia $>120 / \mathrm{min}$ & Yes & No & No & No & No & No & No & No & No \\
\hline \multicolumn{10}{|l|}{ Endoscopic procedure } \\
\hline Total time, minutes & 142 & 145 & 110 & 115 & 115 & 95 & 80 & 105 & 65 \\
\hline Anastomosis suture time, minutes & 40 & 65 & 30 & 40 & 30 & 50 & 30 & 30 & 30 \\
\hline Number of stitches, $\mathrm{n}$ & $4+1$ & $7+1$ & $5+1$ & $6+2$ & $5+1$ & $7+2$ & $6+1$ & $6+1$ & $4+2$ \\
\hline \multicolumn{10}{|l|}{ Follow-up } \\
\hline Transit & Yes & Yes (D5) & Yes & Yes & Yes & Yes & Yes & Yes & No \\
\hline Stool consistency & Normal & Diarrhea & Diarrhea & Diarrhea & Normal & Normal & Normal & Normal & NO \\
\hline Weight before procedure, $\mathrm{kg}$ & 28 & 30 & 30 & 31 & 35 & 25 & 29 & 30 & 28 \\
\hline Weight after procedure, $\mathrm{kg}$ & 28 & 29 & 22 & N & 32 & N & 27 & $\mathrm{~N}$ & N \\
\hline Occlusion & No & No & No & No & No & No & No & No & No \\
\hline Sepsis, postoperative day (POD) & No & No & No & $\begin{array}{l}\text { Yes } \\
\text { (POD 12) }\end{array}$ & No & $\begin{array}{l}\text { Yes } \\
\text { (POD 9) }\end{array}$ & No & $\begin{array}{l}\text { Yes } \\
\text { (POD 5) }\end{array}$ & $\begin{array}{l}\text { Yes } \\
\text { (POD 5) }\end{array}$ \\
\hline Death (POD) & No & No & No & $\begin{array}{l}\text { Yes } \\
\text { (POD 13) }\end{array}$ & No & $\begin{array}{l}\text { Yes } \\
\text { (POD 9) }\end{array}$ & No & $\begin{array}{l}\text { Yes } \\
\text { (POD5) }\end{array}$ & $\begin{array}{l}\text { Yes } \\
\text { (POD 5) }\end{array}$ \\
\hline \multicolumn{10}{|l|}{ Autopsy } \\
\hline Peritonitis signs & No & No & No & No data & No & Yes & No & Yes & Yes \\
\hline Adhesions & No & Some & No & " " & No & Yes & No & No & Yes \\
\hline Anastomotic leakage & No & No & No & " " & No & Yes & No & Yes & Yes \\
\hline Local inflammation & No & No & No & " & No & Yes & No & Yes & Yes \\
\hline
\end{tabular}

associated with bladder pressure measurement. It was considered that the urological procedure would not have any effect on the physiologic growth of this control group.

\section{Euthanasia and histological assessment}

Euthanasia was performed after 21 survival days by lethal injection of potassium chloride with animals under general anesthesia. In the case of death during the 3-week period, necropsies were performed to determine whether signs of anastomotic leakage or peritonitis were present. Before euthanasia, the peritoneal cavity was inspected after laparotomy for signs of peritonitis, and all organs were macroscopically examined for signs of infection, scar formation, and necrosis. Anastomotic patency was assessed by catheterizing the afferent and efferent loops with surgical clamps and a finger respectively. Then the entire anastomosis was removed for histopathological examination, and the diameter of the stoma on the gastric side was directly measured to confirm patency.

The anastomotic healing pattern was assessed by histological analysis showing a fusion of the small-intestinal mucosal and muscular layer against the gastric mucosa and muscular layer, respectively. To assess its integrity, the anastomotic site was also histologically observed for the presence of scar formation, necrosis, inflammation, and fistula.

\section{Statistical analysis}

Descriptive, statistical analysis of normally distributed variables is expressed as means with standard deviations (SDs) and ranges. Qualitative variables are expressed as percentages. The small size of the study did not allow for comparative tests in univariate analysis by Fisher's exact test or the use of a chi-squared distribution to search for predictive factors of death. Unpaired Student's $t$ tests were used to determine the significance of differences between means. A $P$ value $<0.05$ was considered statistically significant. Statistical analysis was performed using the SAS soft- ware (Enterprise Guide 4.1 for Windows, SAS Institute Inc., Cary, NC, USA).

\section{Results}

The data related to technical and clinical outcome from NOTES gastrojejunal anastomosis are summarized in $\bullet$ Table 1.

\section{Endoscopic procedure}

A gastrojejunal anastomosis with a pure NOTES approach was successfully performed in nine animals with an excellent reproducibility. The mean $( \pm$ SD) operative time was $108 \pm 26$ minutes (range 65-142) and most procedures (7/9) lasted less than 120 minutes. The average duration of each step of the procedure was as follows ( $\bullet$ Fig. 7): (i) gastric incision, $9.1 \pm 5.4$ minutes (4-20); (ii) transgastric peritoneal cavity access and jejunal loop selection and transfer, $9.7 \pm 6.3$ minutes (5-25); (iii) Biliary SEMS placement for bridging, $22 \pm 17$ minutes ( $5-55$ ); (iv) jejunal loop incision, $8 \pm 3.5$ minutes ( $5-15)$; (v) anastomotic suture, $38.3 \pm 12.2$ minutes (30-65).

Four to seven sutures were required for the completion of the GJA (mean $5.55 \pm 1.30$ ). Two additional stitches were required for closing the residual gap between the approximated tissue following removal of the biliary SEMS. The pyloric closure procedure required one or two T-tag sutures.

\section{Intraoperative adverse events}

For the duration of anesthesia, none of the animals developed hypoxemia $\left(\mathrm{SaO}_{2}<90 \%\right)$. Regarding hemodynamic stability, one animal developed an episode of supraventricular tachycardia (>120/min) that resolved within minutes without intervention. Needle decompression of the pneumoperitoneum was not required in any of the animals. 


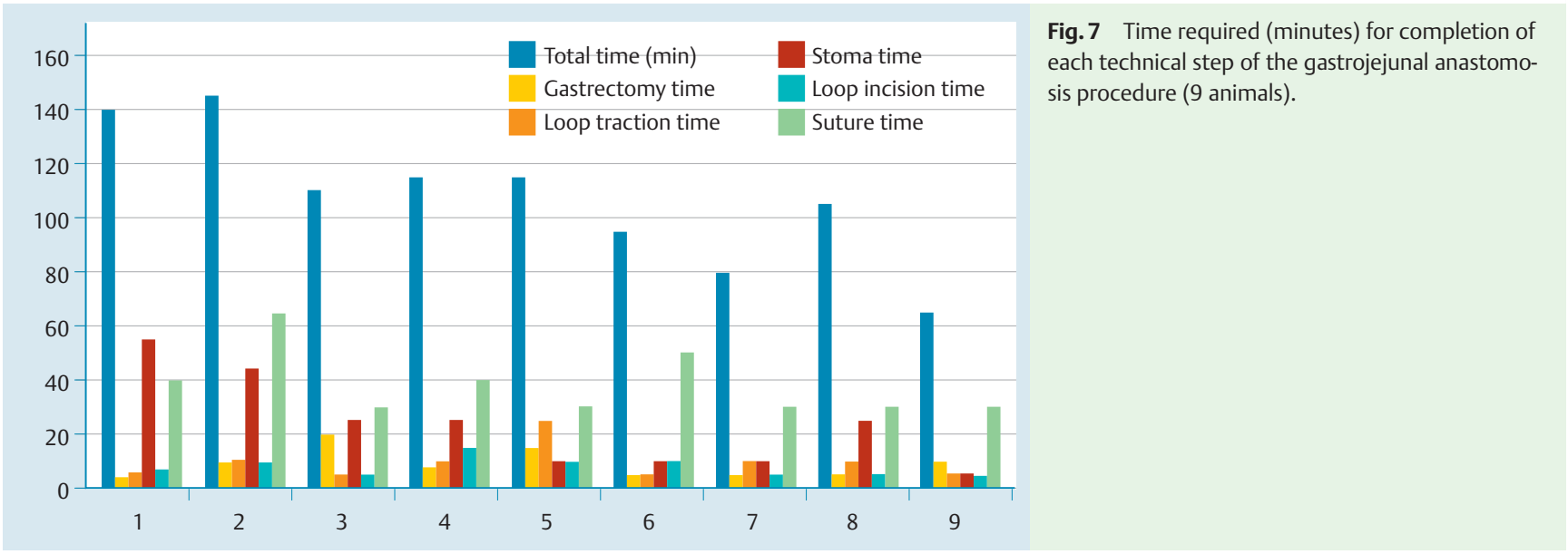

There were no intraoperative adverse events such as bleeding, perforation or organ damage. At necropsy, we did not find any evidence of bleeding or clot formation.

\section{Postoperative outcome and follow-up Clinical course}

In total, five animals had a favorable postoperative course without sequelae. The animals resumed feeding and three animals developed diarrhea. Four animals died due to anastomotic leakage, which was confirmed by the presence of clinical sepsis associated with anastomotic dehiscence and peritonitis confirmed at necropsy. Two of these animals died before POD 5 and the other two before POD 13. The deceased pigs had 5, 7, 9, and 6 stitches in their anastomosis and the average endoscopic duration time was 95 minutes versus 118 minutes for the rest of the animals (not significant [n.s.]). Upon endoscopic inspection, all four animals with anastomotic leaks postoperatively appeared to have shown a satisfactory anastomotic pattern intraoperatively.

\section{Postoperative weight curve}

The weight curve in the five animals that survived in our study was compared with that of the control group. The baseline weight of the current study group was $29.5 \mathrm{~kg}$ whereas it was $29.7 \mathrm{~kg}$ in the control group (n.s.) and all the survival pigs were in perfect clinical condition without signs of sepsis and had a normal refeeding process. It was also assumed that the average weight gain of a 4-month-old pig is $\sim 1 \mathrm{~kg}$ per week.

In our series, we observed an average weight loss of $3.2 \pm 3.1 \mathrm{~kg}$ (range $0-8 \mathrm{~kg}$ ), whereas in the control group, we noted an average weight gain of $5.2 \pm 1.6 \mathrm{~kg}$ [ $3-7 \mathrm{~kg}$ ]. This difference was statistically significant $(P<0.0005)$ demonstrating the effectiveness of the intervention with regard to accomplishing weight loss.

\section{Necropsy findings}

At necropsy, none of the animals that had survived over the 3week postoperative period showed any signs of peritonitis, but some adhesions were observed in one animal. All anastomoses were macroscopically healed with easy identification of both the afferent and efferent loops. Additionally, the gastric opening's macroscopic diameter was measured at $\sim 2 \mathrm{~cm}$ so we could easily separately catheterize the two loops during digital examination ( Fig. 8), whereas this was impossible in the dehiscence group because of a complete leakage.

\section{Histological analysis}

In all the survival animals the GJA sites were available for histological examination. The anastomotic opening at the level of the gastric mucosa had a mean diameter of $20 \pm 4.2 \mathrm{~mm}$. At the level of the anastomotic gastroenteric junction, we observed a complete fusion of mucosal and muscular layers with mild to moderately acute and chronic inflammatory changes. These included highly polymorphic granulomatous tissue with infiltration of lymphocytes, rare plasma cells, macrophages, and neutrophils. The submucosa was infiltrated with collagen, fibroblasts and new blood vessels. A foreign-body reaction at the level of the suture was observed in some of the animals ( $\bullet$ Fig. 9 ).

\section{Discussion \\ $\nabla$}

This experimental study confirms the feasibility of performing a GJA using an exclusively NOTES approach with avoidance of laparoscopic assistance. Potential advantages of a pure NOTES gastric bypass without laparoscopic assistance relate to the avoidance of skin incisions with a decreased risk of ventral hernia and wound infections. The first population of patients that may benefit from NOTES gastrojejunostomy are moribund patients with gastric outlet obstruction. Frequently, because of advanced age or significant co-morbidities, including coronary artery disease or chronic heart failure, these patients may not be candidates for general anesthesia. Because of the less invasive character of NOTES, with the potential for performing NOTES GJA with monitored anesthesia care, endoscopic treatment might be appropriate in these patients by avoiding the general anesthesia that laparoscopy would require. The possibility of performing NOTES under monitored anesthesia care has been demonstrated recently $[26,27]$. However, NOTES GJA may prove to be of benefit also in bariatric surgery. Especially obese patients are more prone to wound infection because the decreased perfusion of fat tissue makes this population more prone to infections. Frequently, obese patients have type II diabetes mellitus, which further increases the risk of wound infection. Often, laparoscopic access proves difficult in severely obese patients because of the increased abdominal wall thickness. It is not uncommon that in patients undergoing laparoscopic Roux-en-Y gastric bypass, a conversion to laparotomy is required. Because of the increased intra-abdominal pressure in obese patients, this population is significantly more prone to ventral hernias. We would agree that for now this endoscopic technique of GJA seems more suitable for the treat- 


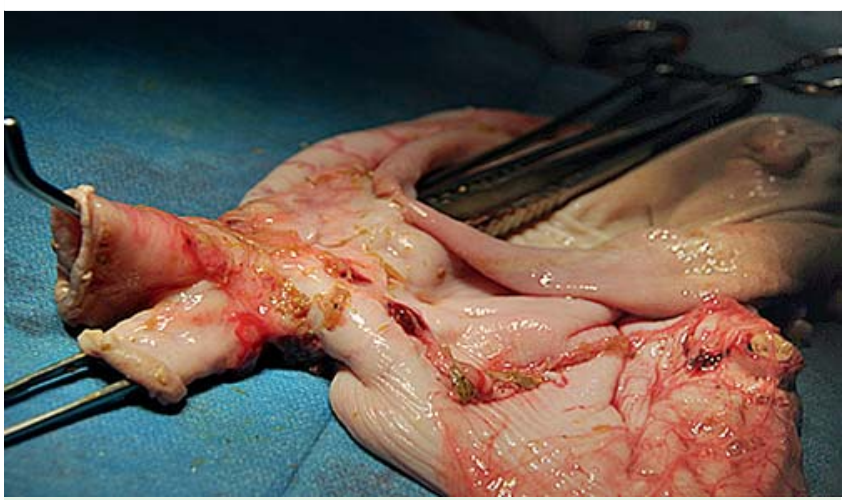

Fig. 8 Macroscopic examination of the gastrojejunal anastomosis during necropsy at 3 weeks post-surgery. Catheterization of each loop with surgical clamps.

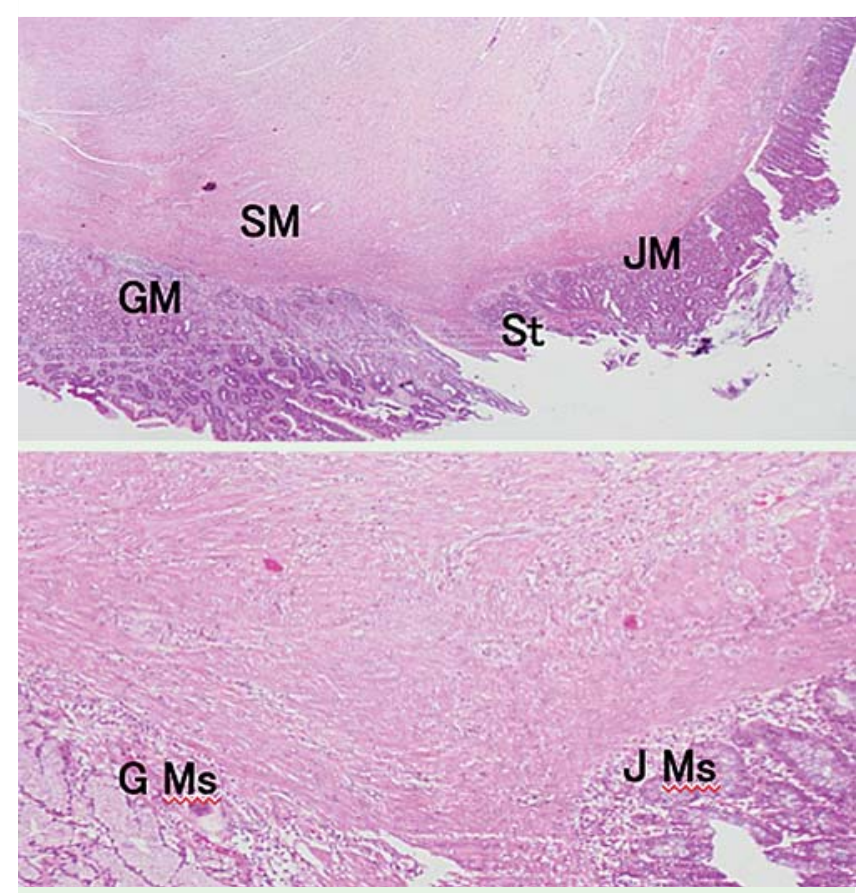

Fig. 9 Histological assessment of the gastrojejunal anastomosis at 3 weeks post-surgery. 1 Apparent scar formation is seen at the level of the mucosa (GM, JM) and beginning of the collagenization of the entire gut wall with presence of discrete inflammatory infiltrate (HES, $\times 1$ magnification). 2 Continuity of muscular mucosa layer is seen at the level of the gut wall presenting with highly polymorphic granulomatous tissue (HES, $\times 1$ 10magnification).

ment of gastric outlet obstruction in a palliative setting. However, in this study, we did not plan a gastric volume-reducing procedure as in sleeve gastrectomies but we did achieve a digestive bypass by associating a GJA and a pyloris closure.

We created a pyloric closure to mimic the conditions seen in benign or malignant gastric outlet obstruction and to create a restrictive component as seen in bariatric bypass procedures. The interventions were performed without any intraoperative adverse events, but we observed postoperative anastomotic dehiscence leading to abdominal infection and sepsis in $44 \%$ (4/9) of the animals. We think that a clinical success rate of $56 \%$ shows that the technique is still a long way from human application, but that with further refinement of the suturing technique the success rate may reach acceptable values. We believe that further technical development is required to improve the T-tag suturing technique to achieve an improved and acceptable survival rate.

The NOTES GJA procedure was also highly reproducible, with little change required. We decided to perform the procedures under air insufflation because $\mathrm{CO}_{2}$ insufflation in animal models for NOTES has not been proved to be better than air insufflation. Effectively, we believe that $\mathrm{CO}_{2}$ would not have made any change on the outcomes. All the stomachs were obviously cleaned prior incision of the gastric wall, as recommended in the literature. In the surviving pigs at the end of the follow-up we did not find any peritonitis. We verified that the incision technique with a simple needle-knife was safe and efficient with results comparable to those of the techniques similar to percutaneous gastrostomy (PEG) described earlier [28]. Spatial orientation in the peritoneum and selection of the jejunal loop were among the most difficult steps in this procedure. Alternative methods have been described for jejunal loop selection but in our opinion they are relatively cumbersome. Kantsevoy et al. describe a tracking method using insertion of an optic fiber into the jejunum via a colonoscope. This procedure requires specialized equipment and further logistical support [29]. Another option could be the use of fluoroscopy for detection of the proximal jejunal loop by contrast injection in the duodenum. Thus, we agree that making a reliable loop selection was a limit in our technique, although this initial step is very important in the standardization of the GJA procedure. In this study, we tried only to select a loop in the middle of the accessible bowel and we are currently working on a study aimed at reliable determination of the length of the selected loop.Another challenge was stabilizing the jejunal loop within the gastrostomy site. In the literature, many techniques for addressing this challenge have been reported, often describing a "hybrid" technique coupled with laparoscopy. Chiu et al. [15] demonstrated a simple grasping technique using forceps prior to suturing with a surgical stapler via both percutaneous and transgastric access. Bergström et al. [11] and Kantsevoy et al. [12] proposed an exposure with a polypectomy loop. The latter group first sutured the jejunal loop to the gastric wall before opening it. The use of a PEG technique has also been demonstrated but this appeared to complicate the procedure and lengthen the examination time [17]. An alternative technique of endoscopic gastroenteric anastomosis using a magnet inserted into the distal duodenum has been recently proposed, with reported efficacy [30]. However, this technique requires several endoscopies and has complication rates that are currently not acceptable. In the present study, we decided to expose the jejunal loop using a "bridge support" technique by inserting a metal stent. This technique is comparable to the creation of a surgical ileostomy using a bridging rod across the mesentery [31]. The suture remains the major challenge in all studies proposing the creation of an anastomosis. We made the GJA using the innovative Brace Bar prototype system, which uses the principle of "sewing" with T-tags using a single needle. This device provided a classic full-thickness suture, with approximation of gastric mucosa to jejunal serosa as confirmed by histological analysis. The limitation of this technique was the prolonged procedure length resulting from the time required to reload the T-tags into the needle after each stitch. Most importantly, we observed incomplete anastomosis as described above.

The clinical follow-up permitted confirmation of the integrity, functionality, and effectiveness of the gastric bypass in the five surviving animals, including the two animals that had a partial gastric closure (pigs \#1 and \#2). Transit was modified in three of 
these animals, suggesting a functional anastomosis. In all these animals, the GJA was perfectly healed at laparoscopic examination, without peritonitis or leakage signs. Most important, for the first time in a GJA animal study we measured and compared the weight curve of the intervention group with that of a control group. We noted an average weight loss of about $3 \mathrm{~kg}$ with a normal feeding pattern, whereas the control group showed an average weight gain of $5 \mathrm{~kg}$ within the 3 -week study period. Pyloric closure was surely one of the explanations for such a weight difference. The size of the gastrojejunal orifice ranged between 12 to $25 \mathrm{~mm}$ suggesting good functionality. We think that the use of the metallic stent helped to accomplish this adequate opening size. The stent allowed better exposure, enabling a larger gastric incision and wider stitch placement around the jejunal loop. Using a hybrid technique with a stapler, Chiu et al. [15] achieved an average orifice size of $30 \mathrm{~mm}$ in 11 animals undergoing gastrojejunostomy, but the group did not assess weight gain or loss postoperatively.

Histological analysis revealed scarring often accompanied by local acute and chronic inflammatory infiltrates at the anastomotic site. Additionally, we also observed an increase in the collagen matrix of junctional zones, especially in the submucosa, and the restoration of the continuity between the mucosal muscle layers. The restoration of the muscular layer occurred more irregularly. In one study, anastomotic healing after a NOTES gastrostomy procedure [32] on 12 pigs showed similar results at 2 weeks post-surgery. Corroborating the findings of other studies [5,33], survival at 3 weeks post-surgery in the present study was primarily related to the strength of the anastomosis.

In conclusion, the results of our study demonstrate the feasibility and efficacy of performing gastrojejunal bypass using an exclusively endoscopic transgastric approach. However, the safety of the procedure was not satisfactory: the high mortality encountered remains a problem of great concern especially as the mortality resulted exclusively from anastomotic leaks. Because of the limited number of animals in this study, we could not establish predictive factors for death from anastomotic leakage. However, some factors known to promote anastomotic adverse events such as anastomotic tension and trauma induced during the procedure are probably not yet fully controlled $[30,34]$. Moreover, in the present study we speculated that leakage was due to the pressure exerted on the healing anastomosis after the pylorus had been closed; such a pressure was higher than the sutures could withstand. We think that in effect the anastomosis may require sufficient time for healing and consolidation before the gastric outlet obstruction is instituted. Based on this assumption, we propose that a subsequent study should investigate the bypass procedure in two steps, with performance of a GJA first and then later the pyloric closure. Obviously, in patients, all the procedures should be performed with $\mathrm{CO}_{2}$ insufflation to improve the outcomes in terms of tolerance of the pneumoperitoneum and abdominal pain, in the absence of contraindication. Further refinement of the suturing technique or modifications of the creation of the anastomosis (by using stents) may help to improve the morbidity and mortality associated with this approach, in addition to using the two-steps approach. Finally, we need to compare our technique with the laparoscopic technique, which is the gold standard, and to evaluate the consequences on metabolic markers following such a NOTES approach.

Competing interests: None.
Institutions

${ }^{1}$ Department of Gastroenterology, Public Assistance Hospitals of Marseille, North Hospital, Marseille, France

${ }^{2}$ Department of Gastroenterology, Beth Israel Deaconess Medical Center, Harvard Medical School, Boston, Massachusetts, United States

${ }^{3}$ Department of Endoscopy, University Hospital of Nice, Nice, France

${ }^{4}$ Aix-Marseille University, CERC, Faculty of Medicine, Marseille, France

${ }^{5}$ Department of Digestive Surgery, Public Assistance Hospitals of Marseille,

Marseille, France

\section{References}

1 Dray X, Marteau P. [The future of gastrointestinal therapeutic endoscopy: NOTES]. Gastroenterol Clin Biol 2009; 33: 758 - 766

2 Giday SA, Kantsevoy SV, Kalloo AN. Principle and history of Natural Orifice Translumenal Endoscopic Surgery (NOTES). Minim Invasive Ther Allied Technol 2006; 15: 373-377

3 Ly J, O'Grady G, Mittal A et al. A systematic review of methods to palliate malignant gastric outlet obstruction. Surg Endosc 2010; 24: 290 297

4 Bakkevold KE, Kambestad B. Morbidity and mortality after radical and palliative pancreatic cancer surgery. Risk factors influencing the shortterm results. Ann Surg 1993; 217: 356-368

5 Jeurnink SM, Steyerberg EW, van Hooft JE et al. Surgical gastrojejunostomy or endoscopic stent placement for the palliation of malignant gastric outlet obstruction (SUSTENT study): a multicenter randomized trial. Gastrointest Endosc 2010; 71: 490-499

6 Maetani I, Tada T, Ukita T et al. Comparison of duodenal stent placement with surgical gastrojejunostomy for palliation in patients with duodenal obstructions caused by pancreaticobiliary malignancies. Endoscopy 2004; 36: $73-78$

7 Brolin RE. Update: NIH consensus conference. Gastrointestinal surgery for severe obesity. Nutrition 1996; 12: 403-404

8 Salameh JR. Bariatric surgery: past and present. Am J Med Sci 2006; 331: 194-200

9 Buchwald H, Avidor Y, Braunwald E et al. Bariatric surgery: a systematic review and meta-analysis. JAMA 2004; 292: 1724-1737

10 Podnos YD, Jimenez JC, Wilson SE et al. Complications after laparoscopic gastric bypass: a review of 3464 cases. Arch Surg 2003; 138: 957-961

11 Bergström $M$, Ikeda $K$, Swain $P$ et al. Transgastric anastomosis by using flexible endoscopy in a porcine model (with video). Gastrointest Endosc 2006; 63: 307-312

12 Kantsevoy SV, Jagannath SB, Niiyama $H$ et al. Endoscopic gastrojejunostomy with survival in a porcine model. Gastrointest Endosc 2005; 62: $287-292$

13 De Palma GD, Persico F, Masone $S$ et al. Natural orifices transluminal endoscopic surgery (NOTES): an overview of technical challenges and complications of transgastric procedures in anesthetized pigs. Minerva Chir 2008; 63: 261-268

14 Simopoulos C, Kouklakis G, Zezos P et al. Peroral transgastric endoscopic procedures in pigs: feasibility, survival, questionings, and pitfalls. Surg Endosc 2009; 23: 394-402

15 Chiu PW, Wai Ng EK, Teoh AY et al. Transgastric endoluminal gastrojejunostomy: technical development from bench to animal study (with video). Gastrointest Endosc 2010; 71: 390-393

16 von Renteln D, Vassiliou MC, McKenna D et al. Endoscopic vs. laparoscopic gastrojejunostomy for duodenal obstruction: a randomized study in a porcine model. Endoscopy 2012; 44: $161-168$

17 Mintz Y, Horgan S, Savu MK et al. Hybrid natural orifice translumenal surgery (NOTES) sleeve gastrectomy: a feasibility study using an animal model. Surg Endosc 2008; 22: 1798-1802

18 Ramos AC, Zundel N, Neto MG et al. Human hybrid NOTES transvaginal sleeve gastrectomy: initial experience. Surg Obes Relat Dis 2008; 4: 660-663

19 Madan AK, Tichansky DS, Khan KA. Natural orifice transluminal endoscopic gastric bypass performed in a cadaver. Obes Surg 2008; 18: $1192-1199$

20 Hagen ME, Wagner OJ, Swain P et al. Hybrid natural orifice transluminal endoscopic surgery (NOTES) for Roux-en-Y gastric bypass: an experimental surgical study in human cadavers. Endoscopy 2008; 40: $918-$ 924

21 Romanelli JR, Desilets DJ, Chapman CN et al. Loop-anchor purse-string closure of gastrotomy in NOTES(R) procedures: survival studies in a porcine model. Surg Innov 2010; 17: $312-317$ 
22 Romanelli JR, Desilets DJ, Earle DB. Natural orifice transluminal endoscopic surgery gastrotomy closure in porcine explants with the Padlock-G clip using the Lock-It system. Endoscopy 2010; 42: 306-310

23 Park PO, Bergström M, Rothstein R et al. Endoscopic sutured closure of a gastric natural orifice transluminal endoscopic surgery access gastrotomy compared with open surgical closure in a porcine model. A randomized, multicenter controlled trial. Endoscopy 2010; 42: 311 - 317

24 von Renteln D, Schmidt A, Vassiliou MC et al. Natural orifice transluminal endoscopic surgery gastrotomy closure with an over-the-endoscope clip: a randomized, controlled porcine study (with videos). Gastrointest Endosc 2009; 70: 732 - 739

25 Vegesna A, Korimilli A, Besetty $R$ et al. Endoscopic pyloric suturing to facilitate weight loss: a canine model. Gastrointest Endosc 2010; 72: 427-431

26 von Delius S, Sager J, Feussner H et al. Carbon dioxide versus room air for natural orifice transluminal endoscopic surgery (NOTES) and comparison with standard laparoscopic pneumoperitoneum. Gastrointest Endosc 2010; 72: 161 - 169 [e1-e2]

27 Lee SH, Kim SJ, Lee TH et al. Human applications of submucosal endoscopy under conscious sedation for pure natural orifice transluminal endoscopic surgery. Surg Endosc 2013; 27: 3016-3020 [Epub ahead of print]
28 Schomisch SJ, Furlan JP, Andrews JM et al. Comparison of anterior transgastric access techniques for natural orifice translumenal endoscopic surgery. Surg Endosc 2011; 25: 3906 - 3911

29 Kantsevoy SV, Niiyama H, Jagannath SB et al. The endoscopic transilluminator: an endoscopic device for identification of the proximal jejunum for transgastric endoscopic gastrojejunostomy. Gastrointest Endosc 2006; 63: 1055-1058

30 van Hooft JE, Vleggaar FP, Le Moine $O$ et al. Endoscopic magnetic gastroenteric anastomosis for palliation of malignant gastric outlet obstruction: a prospective multicenter study. Gastrointest Endosc 2010; 72: $530-535$

31 Sheridan $C B$, Zyromski $N$, Mattar $S$. How to always do a safe anastomosis. Contemp Surg 2008; 64: 68 - 74

32 Dray X, Krishnamurty DM, Donatelli $G$ et al. Gastric wall healing after NOTES procedures: closure with endoscopic clips provides superior histological outcome compared with threaded tags closure. Gastrointest Endosc 2010; 72: 343 - 350

33 van Hooft JE, Uitdehaag MJ, Bruno MJ et al. Efficacy and safety of the new WallFlex enteral stent in palliative treatment of malignant gastric outlet obstruction (DUOFLEX study): a prospective multicenter study. Gastrointest Endosc 2009; 69: 1059-1066

34 Gainant A. [Prevention of anastomotic dehiscence in colorectal surgery]. J Chir (Paris) 2000; 137: 45-50 